\title{
Application of Ambient Analysis Techniques for the Estimation of Electromechanical Oscillations from Measured PMU Data in Four Different Power Systems
}

\author{
Luigi Vanfretti, Luke Dosiek, John W. Pierre, Daniel Trudnowski, \\ Joe H. Chow, Rodrigo García-Valle, and Usman Aliyu
}

\begin{abstract}
The application of advanced signal processing techniques to power system measurement data for the estimation of dynamic properties has been a research subject for over two decades. Several techniques have been applied to transient (or ringdown) data, ambient data, and to probing data. Some of these methodologies have been included in off-line analysis software, and are now being incorporated into software tools used in control rooms for monitoring the near real-time behavior of power system dynamics. In this paper we illustrate the practical application of some ambient analysis methods for electromechanical mode estimation in different power systems. We apply these techniques to phasor measurement unit (PMU) data from stored archives of several hours originating from the US Eastern Interconnection, the Western Electricity Coordinating Council, the Nordic Power System, and time-synchronized Frequency Disturbance Recorder (FDR) data from Nigeria. It is shown that available signal processing tools are readily applicable for analysis of different power systems, regardless of their specific dynamic characteristics. The discussions and results in this paper are of value to power system operators and planners as they provide information of the applicability of these techniques via readily available signal processing tools, and in addition, it is shown how to critically analyze the results obtained with these methods.
\end{abstract}

\section{Index Terms}

Power system oscillations, power system identification, power system parameter estimation, power system monitoring, application of signal processing techniques, synchronized phasor measurements, power system measurements, small-signal stability.

\section{INTRODUCTION AND BACKGROUND INFORMATION}

W ITH the application of advanced signal processing techniques to synchronized phasor measurements it is possible to obtain crucial information about power system dynamic behavior. This information when incorporated in energy management systems can aid in improving the operational reliability of interconnected power systems, and in addition, it may also be used to characterize power system dynamics. With the initiatives carried out in the Western Electricity Coordinating Council [1] for over two decades and others (see references in [2], and [3], [4]), the application of advanced signal processing techniques to power system measurement data has been proven successful for the estimation of mode properties, i.e. mode frequency, damping, and more recently mode shape [5]. Estimates of mode frequencies and damping are indicators of a power systems stress, a declining value of these indicators point to a detriment on the capacity of a power system to operate reliably, and such condition may lead to a system brake up [1]. Mode shape estimates can give a direct indication of the major areas of a power system contributing to the oscillations in a specific mode frequency, and therefore may be used in the future for determining control actions [5]. There are several types of signal processing techniques available to provide these estimates, some are appropriate for transient signals (or ringdown) [6], [7], others are adequate for ambient signals, and others are used when a known probing source is used to excite the power network [8]. Some of these techniques have been implemented in off-line analysis software [9], and are now being incorporated to software tools used in control rooms for monitoring the near real-time behavior of power system dynamics [10].

The techniques used on ambient signals are a subset of signal processing methods for linear prediction, optimum linear filters, and power spectrum estimation which have a relatively long history of their own [11], [12]. The seminal works in [13], [14] introduced the basic assumptions needed to apply these methods to power system data and showed by solving the WienerHopf linear prediction equations that power system modal frequency and damping estimates can be obtained from ambient data only. The fundamental assumption is that there are random changes, mostly comprised of load variations, which excite the small-signal dynamics of a power system. Hence, ambient data analysis can be used to estimate the inherent oscillatory modes of the power system when the main source of excitation of the system modes are random load variations resulting in a low amplitude stochastic time series referred to as "ambient noise" [2].

Under this basic assumption, non-parametric and parametric power spectrum estimation techniques can be used to estimate the power spectrum from synchrophasor measurements (PMU data). In this paper we focus on block-processing algorithms which encompass these two techniques. The first application of block processing is reported in [14]. These algorithms operate on blocks of data, that is, a parcel of continuously-recorded time-synchronized PMU data usually ranging from 5 to 15 minutes. Using a fixed block length a new mode estimate is calculated for each block, all blocks being equally weighted. Updated mode estimates can be calculated as often as necessary, but they will need a block of the most recently measured data. The automated

Original manuscript submitted to the European Transactions on Power Systems, January 2010. Accepted on April 26, 2010.

First minor revision submitted on July 2010. Final revision submitted on August 2010.

L. Vanfretti is with KTH Royal Institute of Technology, Stockholm, Sweden, SE-100 44 (e-mail: luigiv@kth.se)

L. Dosiek and J. W. Pierre are with the University of Wyoming, Laramie, WY 82071 USA (e-mail: ldosiek@uwyo.edu, pierre@uwyo.edu)

Daniel Trudnowski is with Montana Tech, The University of Montana, Butte, MT 59701 USA (e-mail: dtrudnowski@mtech.edu).

J. H. Chow is with Rensselaer Polytechnic Institute, Troy, NY 12180, USA (e-mail: chowj@rpi.edu)

R. García-Valle is with the Technical University of Denmark, Lyngby, Denmark (e-mail: rgv@elektro.dtu.dk)

U. Aliyu is with Abubakar Tafawa Balewa University, Bauchi, Nigeria. (e-mail: uoaliyu@yahoo.com) 
and continuous application of this identification process is known as a "mode meter" algorithm. From the available methods used in block-processing algorithms, here we focus on the use of a non-parametric method, the Welch Periodogram [15], [11] , and a parametric method, the Yule-Walker (YW) method [14], [16]. As a result of applying these techniques and by focusing the analysis in the range of 0.1 to $1 \mathrm{~Hz}$, the electromechanical modes are manifested as visible peaks in power spectrum density estimate (PSD). Narrow peaks in the estimated spectrum indicate light damping, and broader peaks indicate a well damped mode. With any algorithm, there will be estimated modes that are numerical artifacts, and not true system modes. To discriminate between these two results, a "modal energy" method can be used to determine which mode in the intearea mode range has the largest energy in the signal [16], [17].

Spectral estimates may also be used for mode shape estimation; the cross spectral function (CSD) can be used to estimate both the phasing and coherence of the modes among the system generators[18]. As a result, mode shapes can be estimated [5]. Because these algorithms rely on block processing of data windows, they require several minutes of time-synchronized phasor data from different locations in the power network. Mode shape estimation is an active topic of research and is not illustrated in this paper; the reader is referred to [5], [17] for additional information.

Spectral processing techniques have have been successfully applied to characterize the small signal oscillatory modes in the US WECC interconnection [1], [16], the US Eastern Interconnection [19], [20], as well as in the Australian power system [21], [22]. In this paper we present results of the application of non-parametric and parametric methods for mode frequency estimation in several power systems. We have limited our discussion to the application of block processing techniques for mode frequency estimation only (a discussion on mode damping estimation can be found in [16]). In this paper we emphasize the analysis of several hours of synchrophasor measurement data originating from the U.S. Eastern Interconnection (EI), the Western Electricity Coordinating Council (WECC), the Nordic Power System, and FDR data [23] from the Nigerian power system. This paper intends to illustrate how commercially available signal processing tools [24] are readily applicable for PSD estimation (with the pre-requisite that users are knowledgeable of signal processing methods and are familiar with the MATLAB programming language [24]) and that they produce excellent results for estimating power system modes. A second goal of this paper is to share our knowledge on how to critically analyze the results from applying these techniques, and how to deal with several difficulties that may arise when analyzing PMU data. The discussions and results in this paper are of value to power system operators and planners as they provide information of the applicability of these techniques via readily available signal processing tools, and in addition, it is shown how to critically analyze the results obtained with these methods

The remainder of this paper is organized as follows. In Section II we describe the main concepts used for ambient data analysis from a systems perspective. In Sections III, IV, V and VI we provide results from the ambient analysis of archived data from the WECC, the US EI, the Nordic Power System, and from Nigeria, respectively. In Section VII conclusions are drawn, and in the Appendix in Section VIII we provide additional information about the software tools used in this paper.

\section{Mode Estimation from Power System Measurements}

\section{A. Systems Perspective}

Analysis of small signal dynamics assumes that small changes in the power system can be described by a nonlinear differential-algebraic system of equations linearized about an equilibrium [2], i.e.

$$
\begin{aligned}
\dot{\boldsymbol{x}}(t) & =\boldsymbol{A} \boldsymbol{x}(t)+\boldsymbol{B}_{\mathrm{E}} \boldsymbol{u}_{\mathrm{E}}(t)+\boldsymbol{B}_{\mathrm{L}} \boldsymbol{q}(t) \\
\boldsymbol{y}(t) & =\boldsymbol{C} \boldsymbol{x}(t)+\boldsymbol{D}_{\mathrm{E}} \boldsymbol{u}_{\mathrm{E}}(t)+\boldsymbol{D}_{\mathrm{L}} \boldsymbol{q}(t)+\boldsymbol{\mu}(t)
\end{aligned}
$$

where $\boldsymbol{q}$ is an hypothetical random vector perturbing the system, $\boldsymbol{x}$ is the state vector which includes generator angle and speeds, and $t$ is the time. Control actions that can be described as smooth functions of the state $\boldsymbol{x}$ are embedded in the system $\boldsymbol{A}$ matrix. Power system measurements are represented by $\boldsymbol{y}$ which contains measurement noise $\boldsymbol{\mu}$ which is comprised of effects from instrumentation, communication channels, data systems, and other similar devices; generally, measurement noise has a small amplitude. System topology changes may alter the $\boldsymbol{A}$ matrix to various degrees.

The assumption for $\boldsymbol{q}$ is that it is a vector of small-amplitude random perturbations typically conceptualized as noise produced by load switching. It was proposed in [13] that the load switching is primarily integrated stationary Gaussian white noise with each element of $\boldsymbol{q}$ independent. The system can be conceptualized as shown in Fig. 1 where $y_{i}$ is the $i$-th element of $\boldsymbol{y}$ [1]. The MIMO system $\boldsymbol{G}$ is assumed linear. Network topology changes are represented by switches in dynamic gain matrices $\boldsymbol{K}$ and $\boldsymbol{K}^{\prime}$, which may or may not be deliberate. The response of this system can be classified as either transient (also termed ringdown) or ambient. In the case of ambient conditions, the basic assumption is that the system is excited by low-amplitude variations at $\boldsymbol{q}$ and that the variations are typically random in nature. This results in a response at $\boldsymbol{y}$ that is impregnated with the dynamics $G$. The input $\boldsymbol{u}_{\mathrm{E}}$ can be used to inject low level probing signals into the system [8]. A transient response is typically larger in amplitude and is caused by a sudden switch at $s$ or $s^{\prime}$, or a sudden step or pulse input at $\boldsymbol{u}_{\mathrm{E}}$. The resulting time-domain response is a multi-modal oscillation superimposed with the underlying ambient response.

The different types of responses are shown in Fig. 2, which shows a plot of the real-power flowing on a transmission line in the north of the Eastern Interconnection during a system wide disturbance that originated in the Florida Reliability Coordinating 


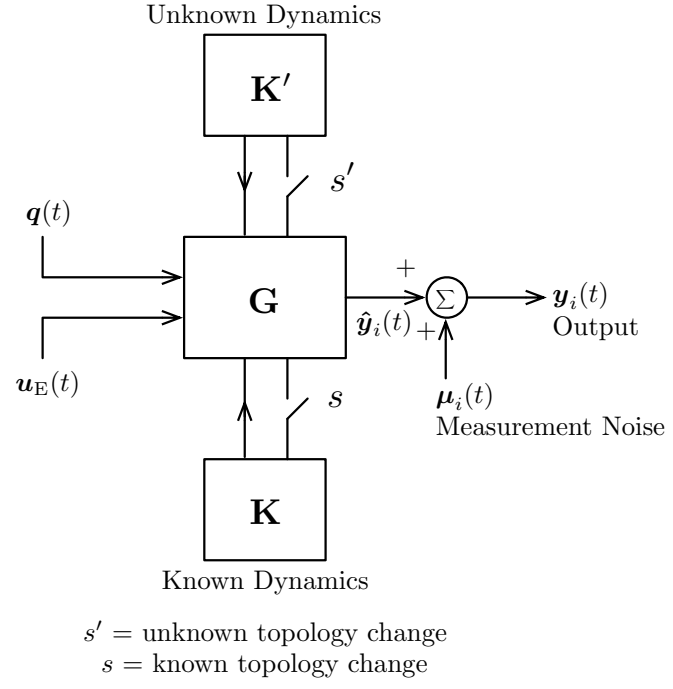

Figure 1. Structure for information sources in process identification [1], [2]

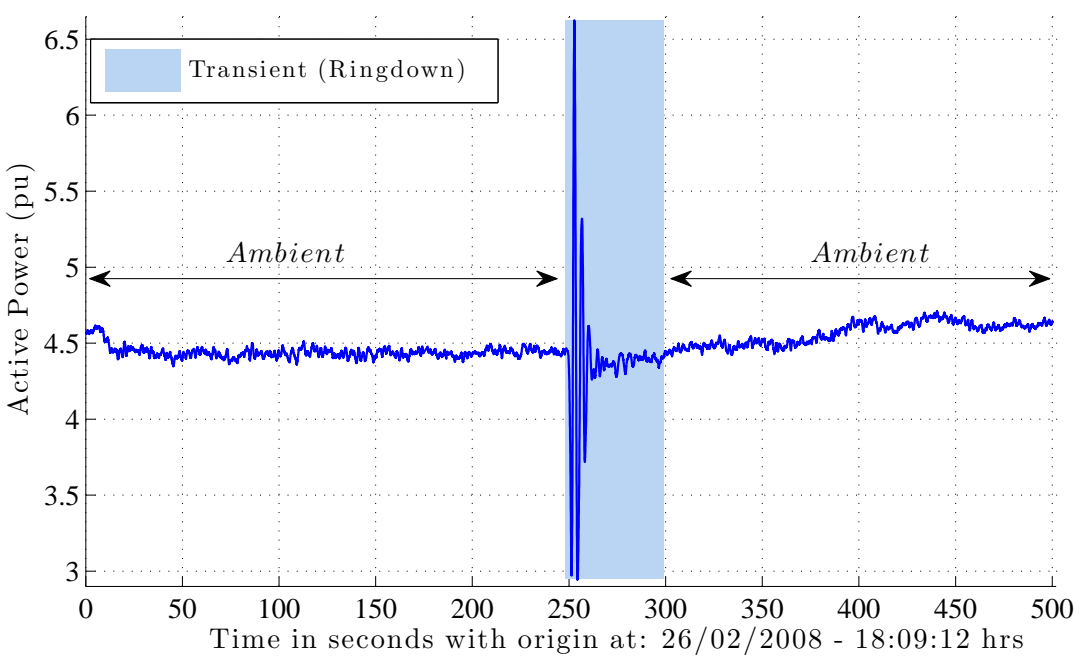

Figure 2. Active power flow on a transmission line in the US EI during the FRCC 2008 disturbance [25]

Council (FRCC) Bulk Electrical System on February 26, 2008 [25]. Prior to the transient that starts at $t=250$ sec., the system is in an ambient condition. The ringdown settles at approximately $t=300 \mathrm{sec}$. and the system returns to an ambient condition.

It must be stressed that because the behavior of of the power system contains a random component, no algorithm can exactly estimate the modal properties. In this paper we analyze ambient data for different power systems, in the next section we briefly describe the two main block processing algorithms used in our analysis.

\section{B. Block Processing Algorithms}

As discussed above block-processing algorithms operate on blocks of data. Using a fixed block length a new mode estimate is calculated for each block, all blocks being equally weighted. Updated mode estimates can be calculated as often as necessary, but they will need a block of the newest data of the same fixed length. To provide these mode estimates, block-processing algorithms implement non-parametric and/or parametric spectral estimation methods.

From the many available methods, here we focus on the use of a non-parametric method, the Welch method [15], [11], and a parametric method, the YW method [14], [16]. The Welch periodogram [11], [15], [26] is a specially robust non-parametric spectral estimation method which gives an estimate of a signal's strength as a function of the frequency; the spectrum is computed using the FFT algorithm. It does not assume an underlaying model, and the estimated spectrum is based on a finite record of data. The algorithm allows for a fairly direct trade-off between frequency resolution (i.e. the ability to resolve closely space frequency components) and the statistical variability of the PSD estimate. The block of data is divided into shorter segments. The FFT of each segment is computed and the squared magnitudes are averaged. This averaging is what reduces the statistical variability in the estimates. The length $N$ of each segment is what determines the frequency resolution $\Delta f=1 /(N T)$ where $T$ is the sampling period ${ }^{1}$. The segments can each be multiplied by a window function to reduce leakage. The segments can also be overlapping to further reduce the variability, but overlapping much beyond $50 \%$ has little extra benefit because of the lack of new data in each piece. The estimated modes are manifested as visible peaks in the power spectrum density estimate. Narrow peaks in the estimated spectrum indicate light damping, and broader peaks indicate a well damped mode or modes that are closely spaced in frequency. Computational requirements of the Welch method can be found in [11].

Even though nonparametric methods are very robust and insightful, because they do not assume an underling model they can not provide numerical estimates of the damping ratio and mode frequency of the modes. On the other hand, parametric methods assume an underlying model whose parameters can be estimated from the measurement data. Once these parameters are estimated, the power density spectrum is computed from the model. As a result, the frequency resolution is much better than the one from nonparametric methods. A commonly used parametric method is the overdetermined modified YW algorithm [14], [16] which is used to estimate the system modes using an autoregressive-moving-average (ARMA) model. Several variations of this method have been proposed in the literature [2]. In this paper the traditional YW algorithm was applied to an autoregressive (AR) model.

The resulting PSDs from these methods are complementary. When the YW method is used to provide mode estimates, the YW spectrum should be compared for consistency with the non-parametric Welch Periodogram spectrum estimate. If a strong

\footnotetext{
${ }^{1}$ For example, given a $10 \mathrm{~min}$. block of data sampled at $f_{s}=5 \mathrm{~Hz}$, segments of $100 \mathrm{sec}$. are typically used. Thus, a frequency resolution of $\Delta f=0.01 \mathrm{~Hz}$ is obtained.
} 
similarity in the spectrum is not observed, the validity of the estimates can be questioned. The two methods discussed in this section are used for mode estimation in the following sections.

By repeating this process on several consecutive overlapping blocks of data, a time-frequency image of the Welch Periodogram can be constructed. We referred to this image as a "Welch spectrogram". When the YW method is used, the time-frequency plot of the AR spectra of the sliding blocks of data is referred to as an "AR spectrogram". These spectrograms provide crucial information about the changing dynamics of power systems.

\section{Mode Estimation FOR THE WECC}

A 24 hour record of ambient data from June 2008 was obtained from PMUs in the WECC. The data was originally sampled at 30 samples per second, preprocessed to remove high frequency noise and low frequency trends, and downsampled to 5 samples per second. At approximately 8.5 hours into the data set, Alberta was disconnected from the rest of the system causing the system dynamics to change. With Alberta connected, there were two well-known North-South modes near 0.26 $\mathrm{Hz}$ and $0.42 \mathrm{~Hz}$. Upon the removal of Alberta from the WECC, the system is expected to be reconfigured to have a single North-South mode near $0.36 \mathrm{~Hz}$.

To demonstrate the insight provided by the analysis of ambient data, frequency data (calculated via differentiating voltage angle data) from a PMU near the northern part of the system was used. The actual name and location of the PMU is hidden to honor confidentiality agreements. Both the Welch and YW methods were applied to the 24 hour data set, which was divided into overlapping 15 minute segments. For each segment, the power spectral density (PSD) was calculated using each of the two methods. The results are shown in the time-frequency plots of Fig. 3. An AR model of order 35 was used.

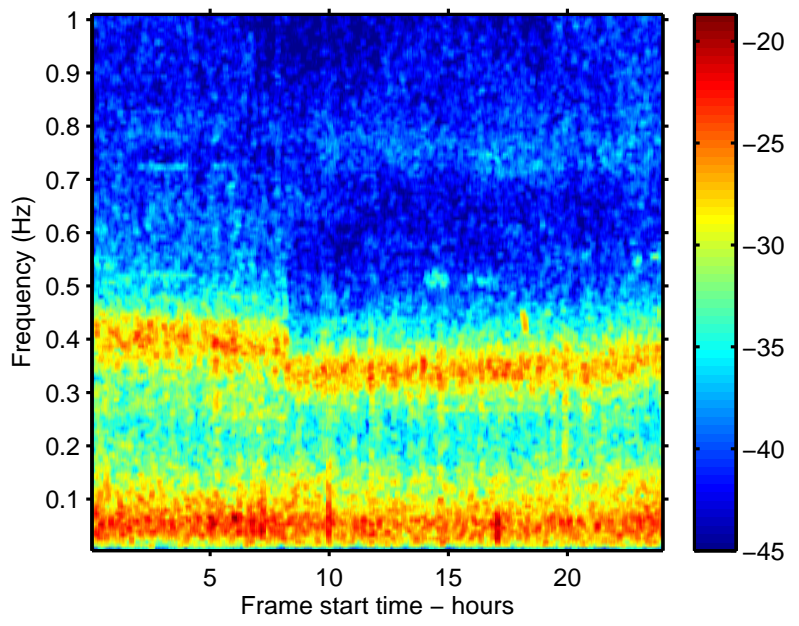

(a) Welch Spectrogram for WECC PMU

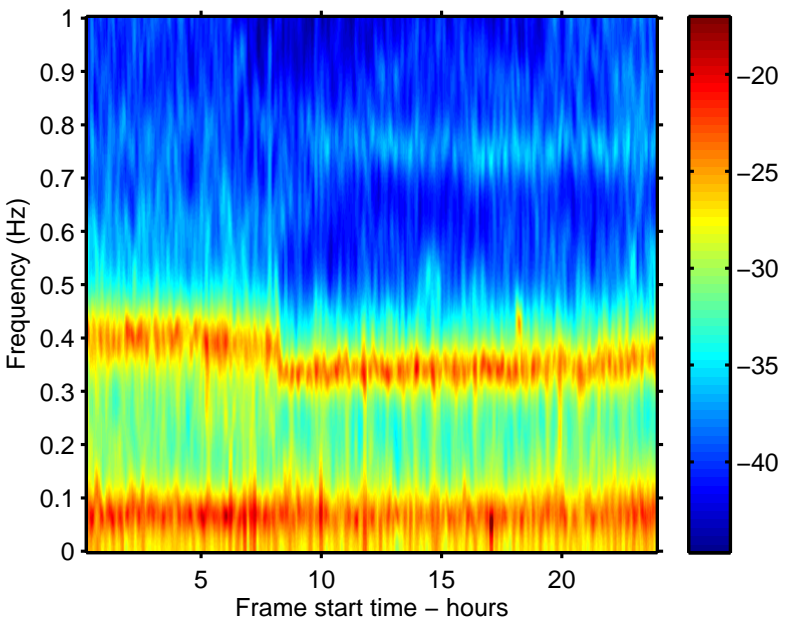

(b) AR Spectrogram for WECC PMU (order 35)

Figure 3. Spectrograms for the frequency data from the PMU of the WECC. The red colors represent maximum values and the blue colors represent minimum values of the power spectrum density $[\mathrm{dB}]$.

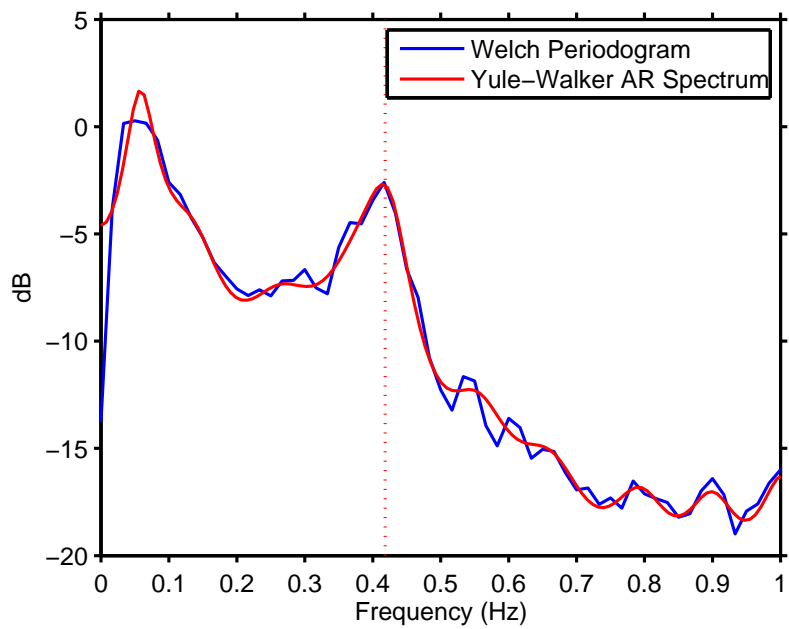

(a) PSD of WECC data with Alberta connected

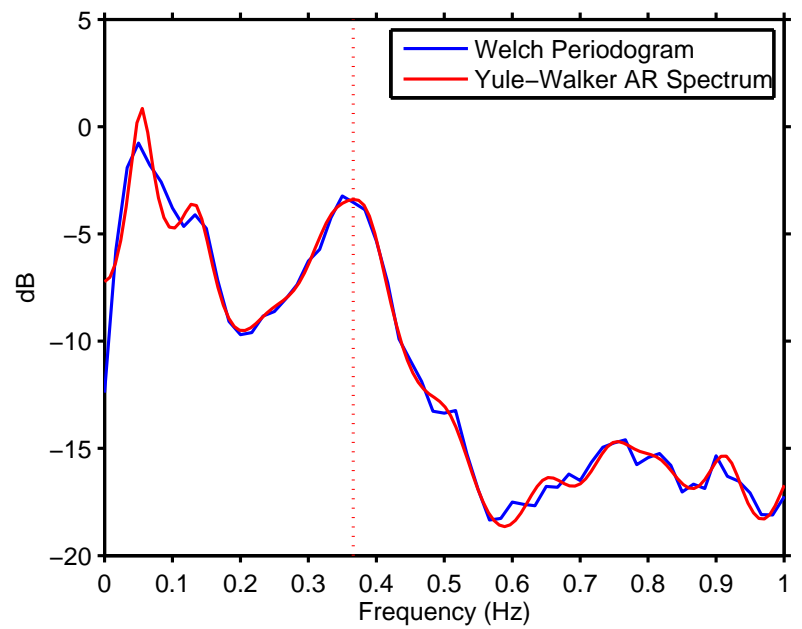

(b) PSD of WECC data with Alberta removed

Figure 4. PSDs of the WECC data showing the shift in modal frequency when Alberta is removed. 
The high energy in the PSDs indicate areas where there is potential for observable electromechanical modes. High energy can also indicate the presence of forced oscillations or very low frequency trends not completely removed in the preprocessing of the data, as seen in the sub $0.1 \mathrm{~Hz}$ areas of Fig. 3. Inspection of the PSDs reveal the obvious system change near 8.5 hours, where the highly observable $0.42 \mathrm{~Hz}$ mode lowers in frequency to $0.36 \mathrm{~Hz}$. Note that the $0.25 \mathrm{~Hz}$ mode is not very observable at this particular PMU. To help interpret the information of Fig. 3, the plots of Fig. 4 were created. Figure $4 \mathrm{a}$ was generated using a single 15 minute segment of data from the time-frequency plots of Fig. 3 during the time when Alberta was connected. Likewise, Fig. 4b was generated using a single 15 minute segment of data from the time when Alberta was removed. Note the decrease in modal frequency is observable in the comparison of Figs. $4 \mathrm{a}$ and 4b. Also note the good agreement between the Welch and YW results. The spectrograms of Fig. 3 are three dimensional plots that contain PSDs for every 15 minute segment of the 24 hour period.

\section{Mode Estimation for the US EASTERn InTERCONNECTiON}

Nearly 19 hours of data from the US Eastern Interconnection (EI) were obtained from PMUs during an event in which most of Florida disconnected from the rest of the system, resulting in a ringdown-type oscillatory response visible throughout the rest of the system. The PMU data was sampled at 30 samples per second and preprocessed in the same fashion as the WECC data. For illustrative purposes, a single PMU was selected in the Northeast portion of the system (the exact name and location hidden) and the same Welch- and YW-based PSD estimation techniques discussed above were applied. The results are presented in Fig. 5.

The Florida Event is observed just after the 12 hour mark as the high energy region that is narrow in the time domain. The area of very low energy just before 5 hours is due to there being a section of missing data. The PSDs over the time of missing data were set to be equal to zero, so in the $\mathrm{dB}$ scale of Fig. 5 they show up as the minimum energy of the dB coloring scale. The area of high energy from approximately $0.18 \mathrm{~Hz}$ to $0.4 \mathrm{~Hz}$ indicates one or more highly damped electromechanical modes. Damping is proportional to the thickness of the energy in the frequency domain; wide band signals imply high damping or closely spaced overlapping modes and narrow band signals imply low damping. Note then the very narrow band signal near $0.45 \mathrm{~Hz}$. At first glance this could be interpreted as an electromechanical mode with dangerously low damping. Further studies suggested that it could actually be a forced oscillation.

The strongest piece of evidence that the energy at $0.45 \mathrm{~Hz}$ is thought to be from a forced oscillation and not a mode is that the frequency of the energy was constant over the course of the day while the amplitude and phasing changed. As the day progressed, subtle changes in the power system due to configuration and loading caused electromechanical modal frequencies to slowly vary with the system changes. These subtle system changes would have contributed to the variable amplitude and phase of a forced oscillation while its frequency would have remained constant.

Also supporting the fact this was an oscillation is that it was observable throughout the EI on the day of the Florida Event in both PMU and FNET data, as well as in PMU data from November 2007 at precisely the same frequency. This was verified by performing a Welch Periodogram on long data segments that used FFT lengths that were approximately a whole number of periods of 1/0.45 seconds. One such periodogram is shown in Fig. 6 where the frequency of the oscillation is estimated to be $0.4539 \mathrm{~Hz}$. When applied to the other data sets, the frequency was always estimated to be $0.4539 \mathrm{~Hz}$.

Lastly, the fact that this phenomenon at $0.45 \mathrm{~Hz}$ is extremely narrow in the frequency domain points to the fact that it is a forced oscillation. Pure sinusoids in the frequency domain are represented as line spectra, that is they have no thickness. This

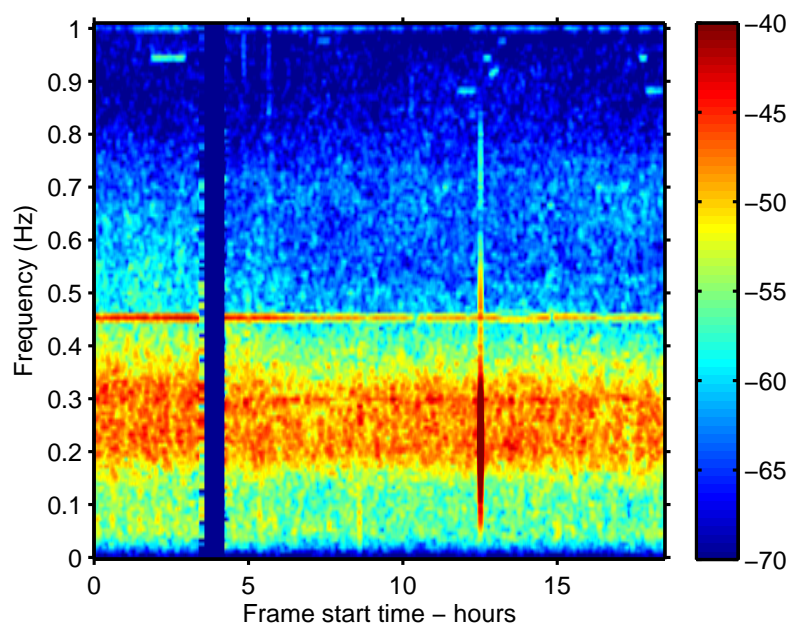

(a) Welch Spectrogram for EI PMU

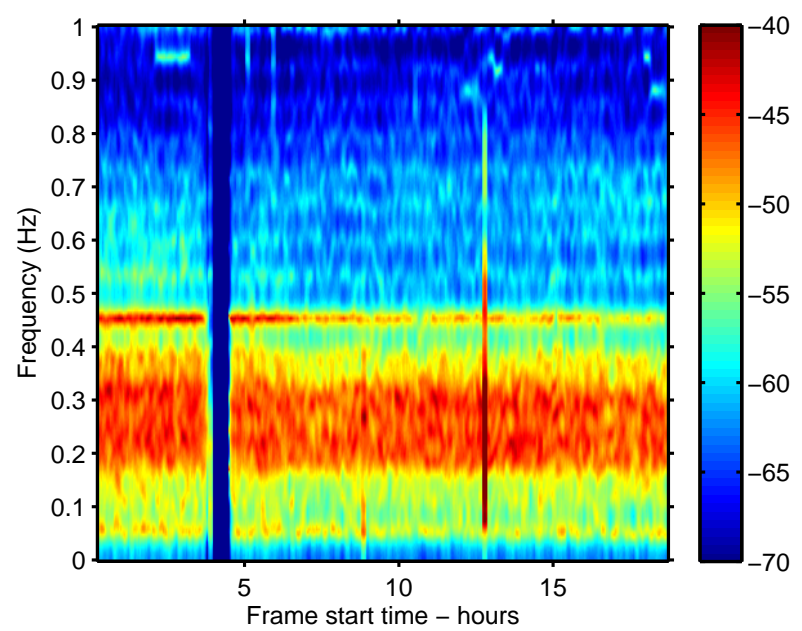

(b) AR Spectrogram for EI PMU (order 75)

Figure 5. Spectrograms for the frequency data from the PMU of the EI. The red colors represent maximum values and the blue colors represent minimum values of the power spectrum density $[\mathrm{dB}]$. 


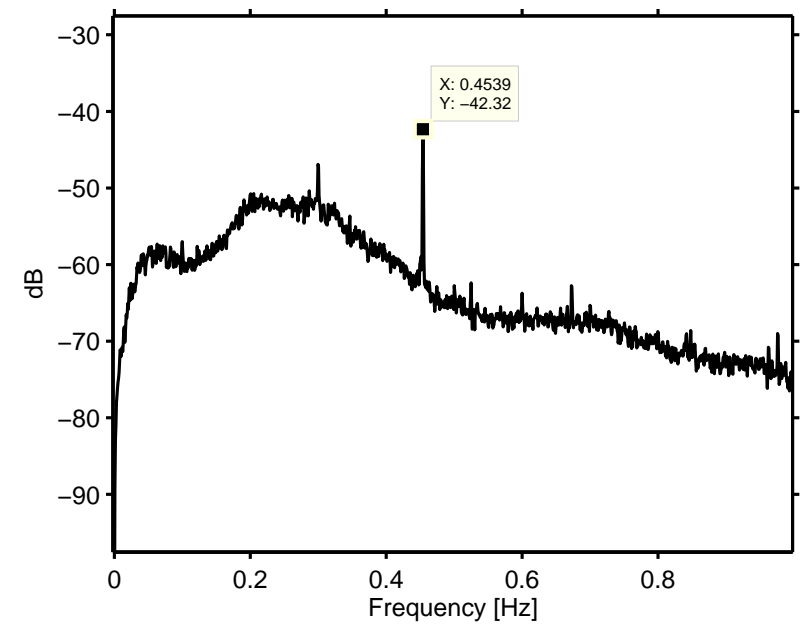

Figure 6. Welch Periodogram of long segment of EI data showing the $0.45 \mathrm{~Hz}$ oscillation

can be interpreted as a signal with $0.0 \%$ damping. This can be difficult to model with a parametric method of low order. For the spectrogram of 5b, the order of the AR model needed to be 75 (as compared to 35 in the WECC data where no forced oscillations were observed) in order for it to begin to match that of the nonparametric Welch periodogram. With the model order sufficiently high, the YW method did in fact begin to model a pole at approximately $0.45 \mathrm{~Hz}$ with $0.0 \%$ damping, further suggesting this was a forced oscillation at $0.45 \mathrm{~Hz}$ and not an electromechanical mode. In [27], oscillations near $0.4 \mathrm{~Hz}$ are reported as being first seen in the eastern system in 1991 and numberous subsequent observations. In all cases, the oscillation was lightly damped. Further analysis is required to confirm that this is the same oscillation observed in more recent data.

\section{Mode Estimation for the Nordic Power System}

In this section PMU data obtained from two different substations of the Nordic power system located in Eastern Denmark is analyzed, their locations are shown in Fig 7. Data from the Radsted substation (RAD132, in Fig. 7) were obtained during 03-20-2008 and 03-21-2008. Data for the Hovegaård substation (HVE400, in Fig. 7) were obtained during 04-15-2007 and 04-16-2007.

The data is segmented in blocks of 10 minutes, and pre-processed for analysis. Active power flow signals are derived from

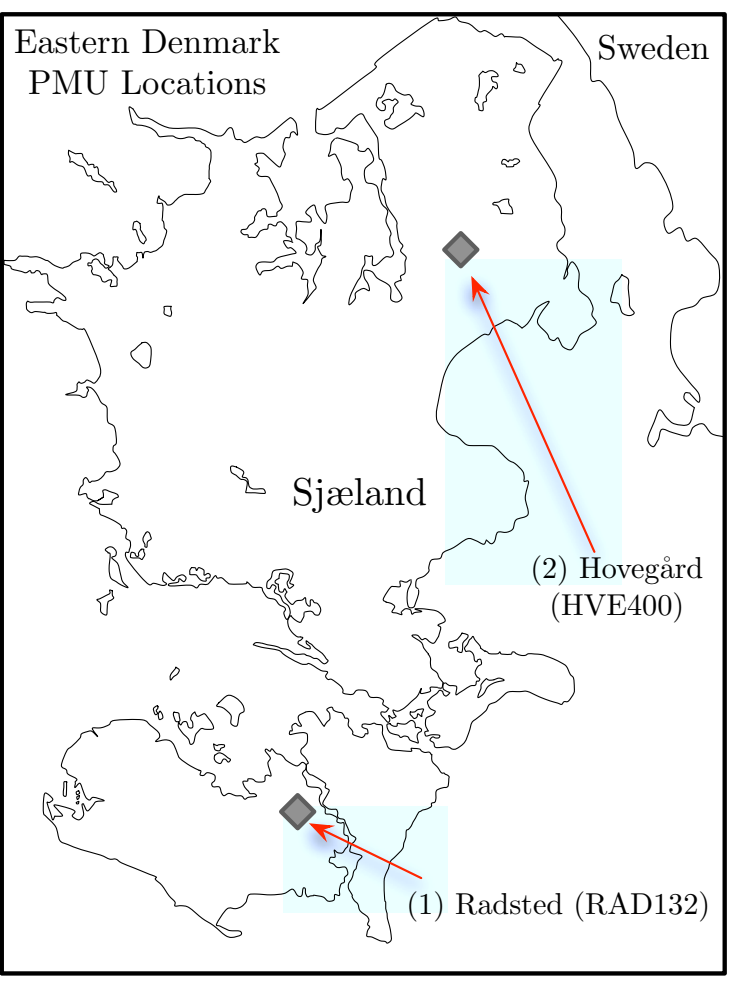

Figure 7. Eastern Denmark PMU Locations

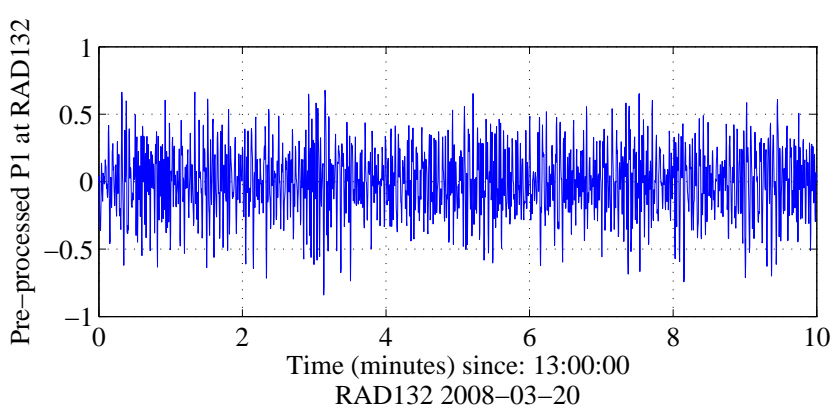

(a) Sample pre-processed $10 \mathrm{~min}$. data block

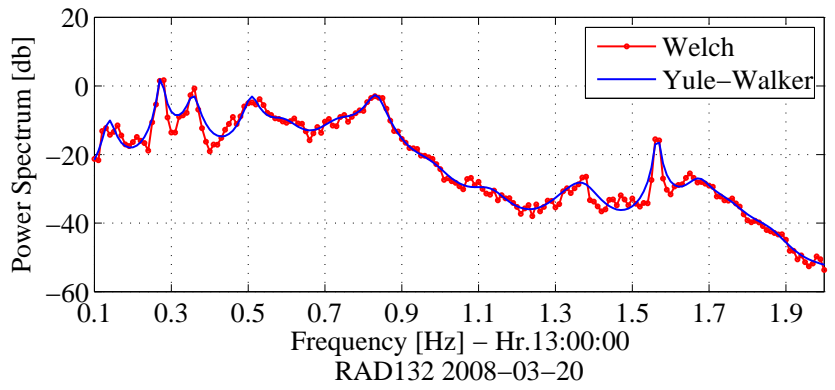

(b) PSDs from the Welch and Yule-Walker Methods

Figure 8. A pre-processed 10 min. data block of the $P_{1}$ signal of Radsted, and its estimated PSDs using the Welch and Yule-Walker methods 
the voltage and current phasors ${ }^{2}$, and bus frequencies are computed by taking the numerical derivative of the voltage angle. First linear trends are removed from these signals using the detrend algorithm. Because these analyses focuses on the inter-area mode range, a low-pass finite impulse response (FIR) filter with $2 \mathrm{~Hz}$ cut-off frequency is used to remove frequency components beyond this cut-off frequency. The data is down sampled to $5 \mathrm{~Hz}$ and a FIR high-pass filter with cut-off frequency of $0.1 \mathrm{~Hz}$ is used to remove frequency components related to governing control. To each $10 \mathrm{~min}$. block of pre-processed data the Welch and YW methods are applied. A summary of the signal processing tools used for these pre-processing steps is given in Section VIII.

First, the Welch method is applied to the pre-processed data to obtain estimated PSDs. We use $100 \mathrm{sec}$ for both the block segments and the number of FFT points used to calculate the PSD. To these segments a Hanning window with 50\% overlap is applied. Fig. 8b shows the estimated periodogram for the $10 \mathrm{~min}$. data block in Fig. 8a. Next, the YW method is applied. The estimated periodograms from Welch's method are used to refine the AR model order of the YW method by obtaining good agreement between the PSDs of both methods, while trying to maintain the model order as low as possible (in this case the order model is $p=40$ ). As a result, an excellent agreement was obtained between the PSDs estimated from each method. In Fig. 8b the YW PSD is shown along with the one obtained by Welch's method. Notice the close agreement between both spectrum estimates.

Subsequently, both methods were applied to all the remaining signals from Radstead and Hovegård for a similar 10 min. block. Note that the estimates for Hovegård were obtained for a different date than those of Radsted. Common to all of the data sets were three dominant modes at approximately $0.36,0.54$, and $0.83 \mathrm{~Hz}$, as indicated by the peaks in the spectra of Fig. 8b. The presence of a low-frequency oscillatory component at approximately $0.277 \mathrm{~Hz}$ is discussed below.

Next a time-frequency analysis for the whole $48 \mathrm{hr}$. data set is performed, and the spectrograms in Fig. 9 were obtained for the $P_{1}$ signal, and in Fig. 10 for the $P_{4}$ signal of Radsted. For the $P_{1}$ signal of Radsted its Welch spectrogram is shown in Fig. 9a, and its corresponding AR spectrogram in Fig. 9b. Observe the close agreement between both spectrograms confirming the existence of the modes discussed above. Similarly, the same modes were confirmed for the $P_{4}$ signal by inspecting the spectrograms in Figs. 10a and Fig. 10b.

The changing dynamics of the power system are revealed by the 48 hrs. spectrograms. Note that 03-20-2008 was a "typical day" (in terms of the power system loading), while 03-21-2008 was a national holiday with decreased loading. It is important to note that the frequency and damping ratio of the electromechanical modes are influenced by the system loading and configuration of the power grid. For example, Mode 2 (around $0.54 \mathrm{~Hz}$ ) is present throughout 03-20-2008, however it is not visible during the 32-42 hrs. segment (9:00 am - 7:00 pm in local time of 03-21-2008), which includes regular weekday working hours, it is likely that during this time frame there was a decrease on the system loading. It is interesting to observe that as a result of the different loading conditions the frequency of Mode 2 varies during hours 0-32 hrs. As similar behavior is observed by Mode 1 (around $0.36 \mathrm{~Hz}$ ), and Mode 3 (around $0.83 \mathrm{~Hz}$ ). The reader might be misled by the "blur" which appears around 24 hrs. in Figs. 9 and 10 which is a result of the selected range of the temperature bar giving the coloring to the spectrogram. To clarify, in Fig. 11 we show an enlargement of Fig. 10a for $t=[16-32]$, centered at $24 \mathrm{hrs}$ and with a different setting

\footnotetext{
${ }^{2}$ For convenience, pre-processed active power flow signals are denoted by $P_{i}$, where $i$ is the line number from where measurements are made.
}

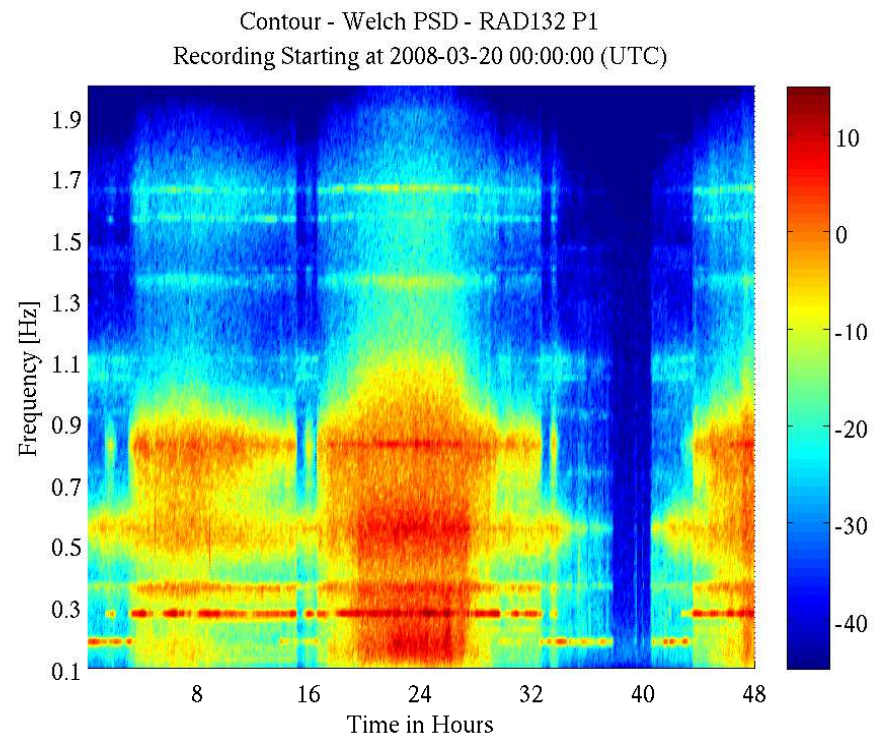

(a) Welch Spectrogram for $P_{1}$

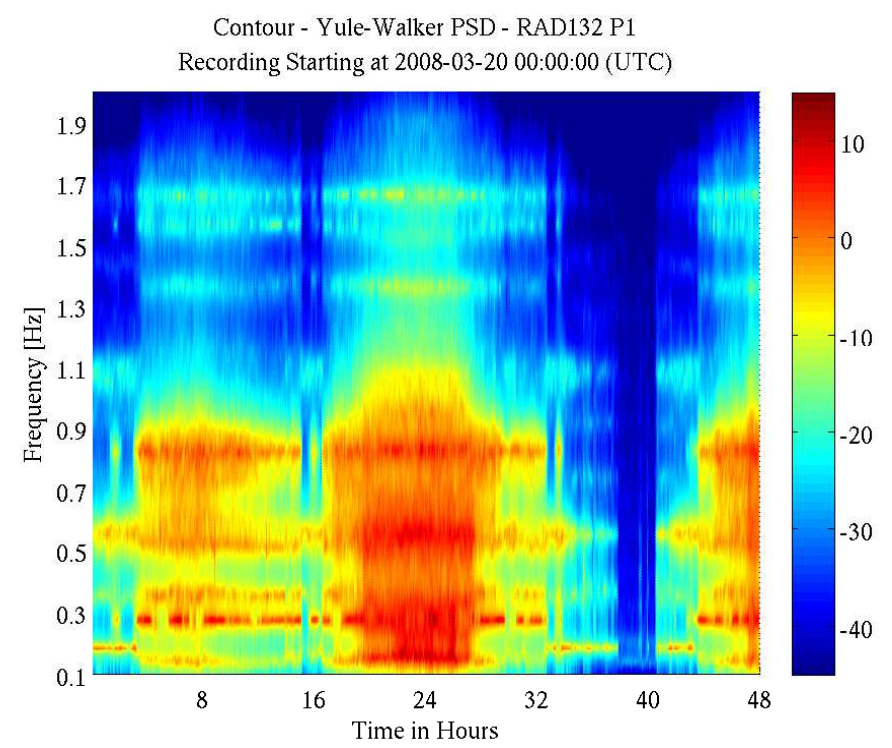

(b) AR Spectrogram for $P_{1}$

Figure 9. Welch and AR Spectrograms for the $P_{1}$ signal from Radsted. The red colors represent maximum values and the blue colors represent minimum values of the power spectrum density $[\mathrm{dB}]$. The time is given in hours in (UTC) starting from 00:00:00 hrs, local time is given in UTC+1 hr. 


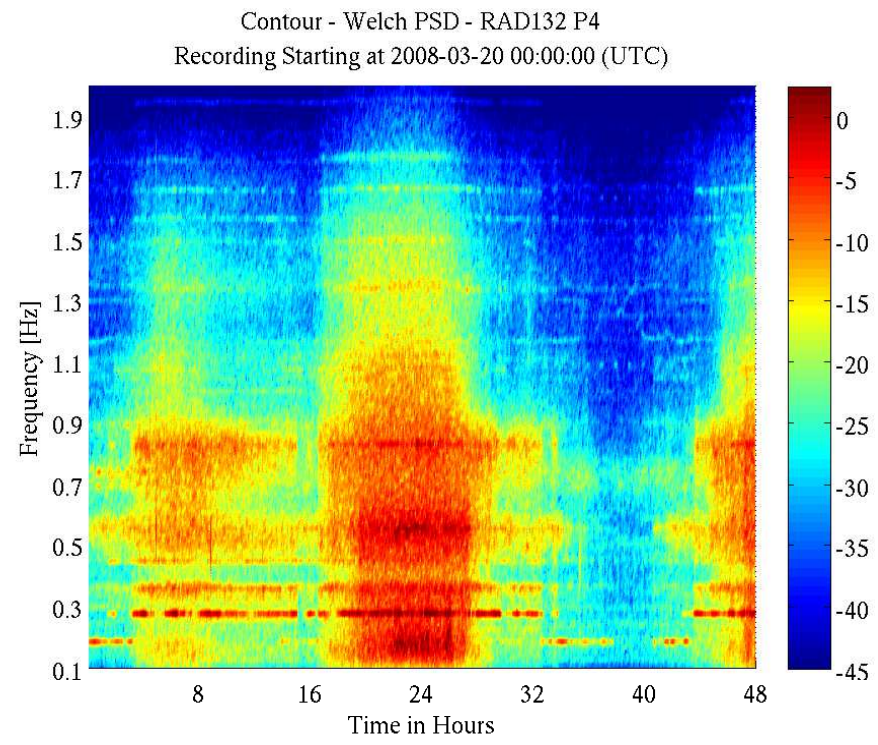

(a) Welch Spectrogram for $P_{4}$

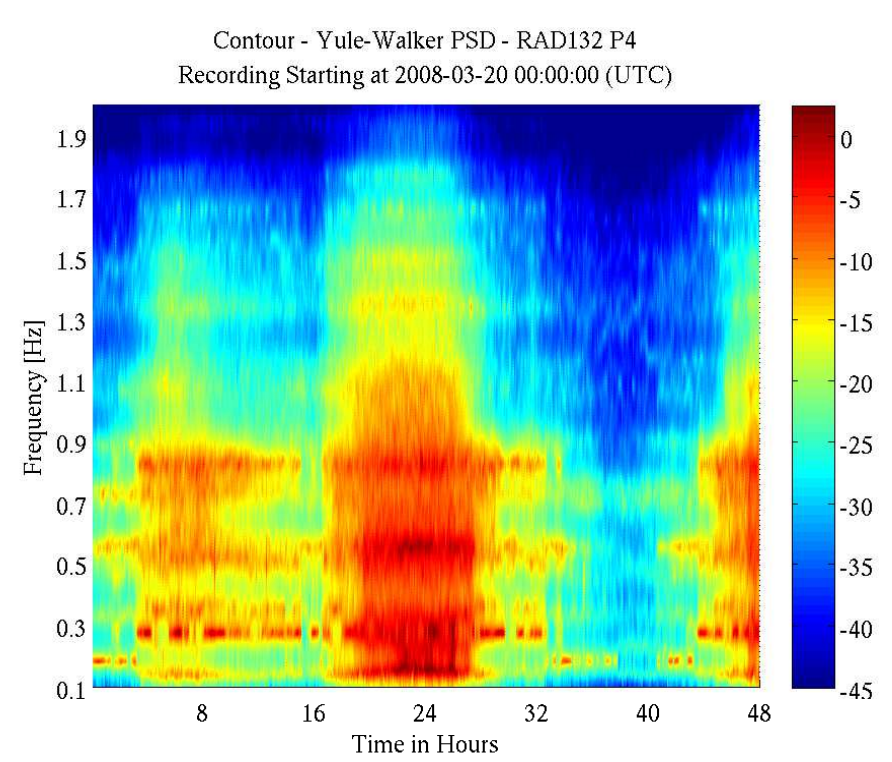

(b) AR Spectrogram for $P_{4}$

Figure 10. Welch and AR Spectrograms for the $P_{4}$ signal from Radsted. The red colors represent maximum values and the blue colors represent minimum values of the power spectrum density [dB]. The time is given in hours in (UTC) starting from 00:00:00 hrs, local time is given in UTC+1 hr.

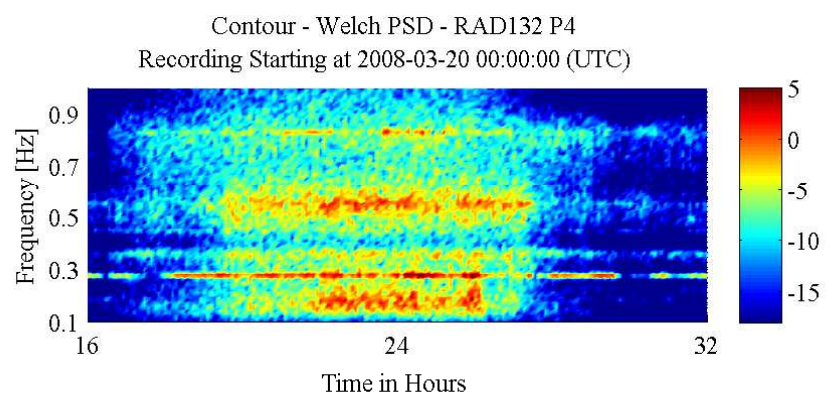

Figure 11. Enlargement of Fig. 10a for $t=[16-32]$, centered at $24 \mathrm{hrs}$. Note that the range for temperature bar has been also modified.

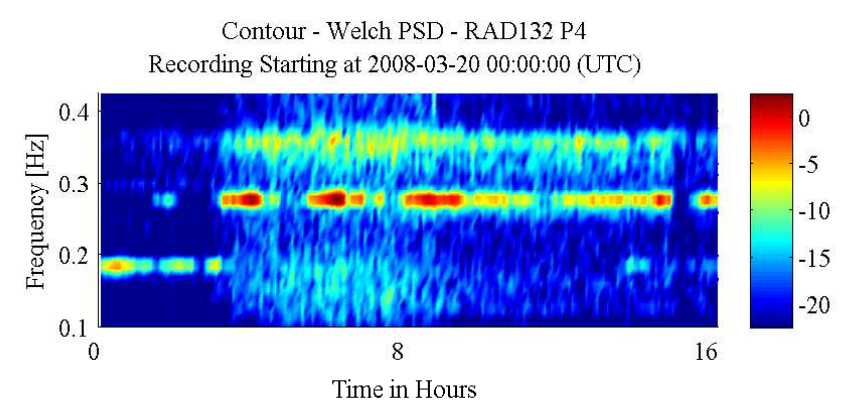

Figure 12. Enlargement of Fig. 10a for $t=[0-16]$ and $f=[0.1-0.4] \mathrm{Hz}$. Note that the range for temperature bar has been also modified.

for the temperature bar. Note that with this new range the "blur" is not longer present and the modes discussed above can be clearly appreciated.

Close inspection of the Welch spectrograms for $P_{1}$ and $P_{4}$ (Figs. 9a, and 10a) reveals an important feature of this particular data set. As mentioned earlier, an oscillatory component is present at about $0.28 \mathrm{~Hz}$. This component must be critically analyzed, and the Welch spectrograms serve to this purpose. The Welch spectrograms show that the $0.28 \mathrm{~Hz}$ component appears almost persistently at a very narrow band, centered at approximately $0.28 \mathrm{~Hz}$, from hour $3-32$ and $42-48$. Also important to note is that the intensity of this component is quite consistent through the time frames mentioned. To further discuss the nature of this component, an enlargement of Fig. 10a is shown in Fig. 12.

Note that from Fig. 12 the behavior of the $0.28 \mathrm{~Hz}$ component is much different from the one observed of Mode 1 where the mode has a broader variation frequency range and change of intensity. By inspecting 12 It becomes apparent that the 0.28 $\mathrm{Hz}$ component has a narrower and better defined frequency band.

The behavior shown by this $0.28 \mathrm{~Hz}$ oscillatory component corresponds to what is expected of a sinusoid or forced oscillation. A more careful inspection of the Welch spectrograms reveals that presumably another sinusoid is present at about $0.18 \mathrm{~Hz}$ for the time time frames of 0-3, 32-37, and 40-43 hrs. A this point, it should be realized that it is very likely that both of these components are harmonics of a fundamental sinusoid of $0.09 \mathrm{~Hz}$. In fact, it is possible to observe traces of other harmonics in Figs. 9a and 10a at $n=2-6,8-13,15-19$ and 21, where $n$ is the number of the harmonic, with corresponding harmonic frequencies of $f_{n}=0.18,0.28,0.37,0.46,0.55,0.74,0.83,0.92,1.02,1.11,1.20,1.39,1.48,1.57,1.66,1.76$ and $1.94 \mathrm{~Hz}$. The origin of the sinusoid and its harmonics is unknown; it could be possibly due to a process in the system (as a control system going into a limit cycle), aliasing from higher-frequencies, or communication or measurements issues. It is important to note that some of the sinusoid harmonics are superimposed over the "true" system modes. Regardless of their origin, these sinusoid harmonics will create difficulties to obtain accurate damping estimates of the true system modes. Mode meter algorithms [16] will have difficulties resolving the portion of the frequency spectrum that is due to ambient load variation from the portion due to the forced oscillations. This difficulty posses a new research challenge to improve the accuracy of mode damping estimates in mode meters. 

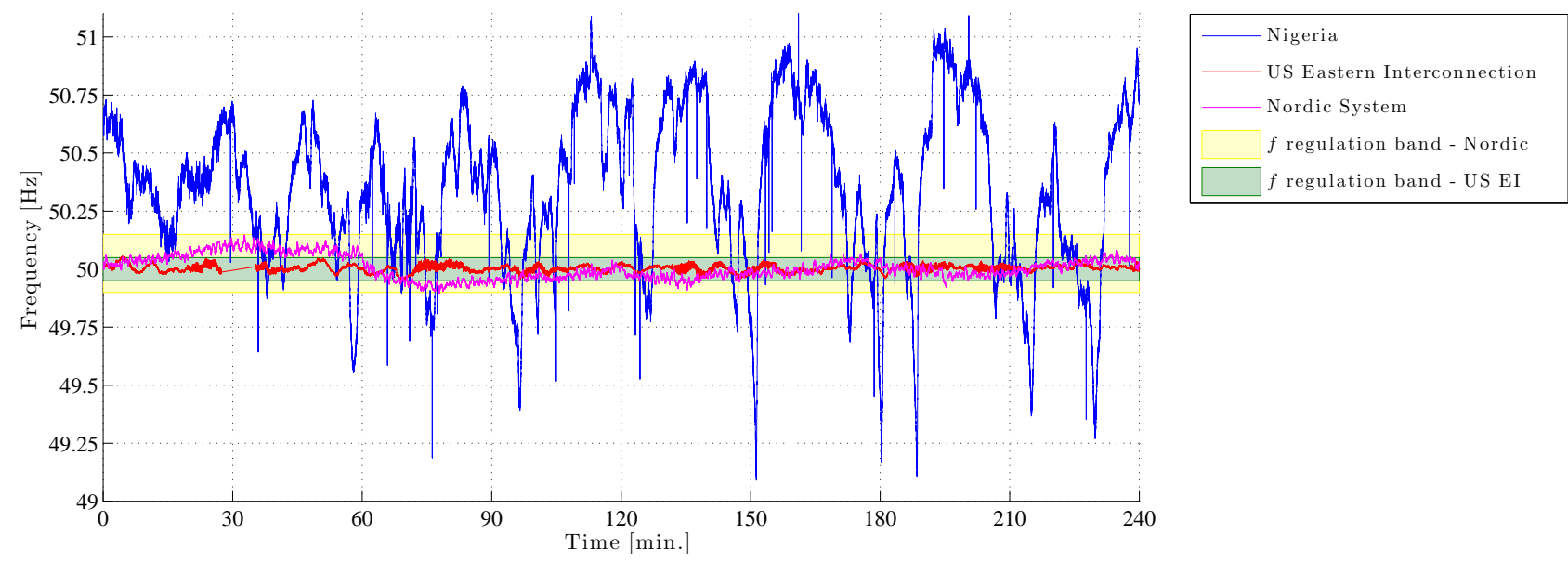

Figure 13. Comparison of the system frequency in different power systems over a 4-hour period. The steady state frequency at the US EI has been shifted by $10 \mathrm{~Hz}$ to coincide with the system frequencies of the Nigerian and Nordic networks.

\section{Mode Estimation for the Nigerian Power System}

\section{A. A Comparison of System Dynamics Observed from Prolonged Recordings}

Differently from the power systems analyzed before, power systems constrained by insufficient generation capacity experience noticeable frequency changes when subject to disturbances or control actions. In these types of systems the primary concern is to maintain the load and generation balance, and therefore, frequency regulation is loose. Consider the frequency measurements taken for a time window of four hours as shown in Fig 13. This plot compares frequency measurements from two different types of power systems: a loosely regulated power system given by the Nigerian power system, and two tightly regulated power networks, the US Eastern Interconnection (EI) and the Nordic Power System. The measurements of the US Eastern Interconnection are derived from the voltage phasor angle of a PMU in the northern part of the EI, the measurements of the Nordic System were derived from the PMU in Radsted substation in Eastern Denmark, and the frequency measurements from Nigeria were obtained from a Frequency Disturbance Recorder (FDR) [23] installed in Bauchi, Nigeria [28].

From these four hour recordings it is observed that the frequency regulation band in the US Eastern Interconnection ranged from 59.95 to $60.05 \mathrm{~Hz}(\Delta f=0.1 \mathrm{~Hz})$, while the frequency regulation band in the Nordic system varied from 49.9 to $50.15 \mathrm{~Hz}(\Delta f=0.25 \mathrm{~Hz})$. Compared with these power systems, the frequency in Nigeria is loosely regulated with variations ranging from 49 to $51 \mathrm{~Hz}$, a $1 \mathrm{~Hz}$ variation from the steady state of $50 \mathrm{~Hz}$. In addition to the upper and lower bounds of frequency variation, it is also important to note that frequency can vary between these bounds in less than 10 minutes, with abrupt frequency changes of $0.4-0.5 \mathrm{~Hz}$ in $1 \mathrm{~min}$. windows. In contrast, both the US EI and the Nordic System have a tightly regulated operation maintaining frequency variations closely between the frequency bands discussed above. Because the frequency variations in these systems are close to their steady state frequency, it is possible to obtain suitable "ambient data" for spectral analysis with standard pre-processing of the measurements as discussed in the previous sections.

\section{B. Data Pre-processing}

Due the loosely regulated nature of the system frequency in the Nigerian grid, it is necessary to first condition the measurement data so that spectral analysis techniques can be applied. In Figure 14 a block diagram of a proposed pre-processing method is shown. Fig. 15 shows the results of applying the method to a 10 min. data block from the Tue, Jan. 23, 2007 (N17) recording.

The method starts by taking the raw frequency measurement from the FDR $(f)$ and removing linear trends from the data by using the detrend algorithm [24]. The result of applying this step to the $10 \mathrm{~min}$. data block is shown in Fig. 15a with a solid blue line ("Detrended Frequency" in the legend). The next step is to apply a high pass filter with cutoff frequency of $0.02 \mathrm{~Hz}$ to the data, this is shown by a red solid line in Fig. 15a ("Filtered $\Delta f$ Signal" in the legend).

By applying this filter to the data the moving average of the frequency, which corresponds to the slowest mode frequencies in the signals (below $0.02 \mathrm{~Hz}$ ), has been effectively removed. In Fig. 15a a magenta dashed line shows the moving average calculated by subtracting the "Detrended Frequency" to the "Filtered $\Delta f$ Signal". The frequency components removed by these steps correspond to the frequency of the process involved in balancing the load and generation in the Nigerian network. With this approach, we have obtained an ambient signal which is mostly comprised by the electromechanical modes of the system.

The final step in the pre-processing method is to remove outliers from the filtered signal. To this aim the mean and standard deviation of the filtered signal are computed. Any point which exceeds the mean by 3.5 standard deviations is removed from the filtered signal. Figure 15b shows the removed outliers in red stems and the filtered signal with outliers removed in solid blue, the later is used for ambient data analysis. Next ambient analysis techniques are applied to prolonged frequency measurements from the Nigerian network, all of the data blocks involved in these calculations are subject to the pre-processing method described above. 


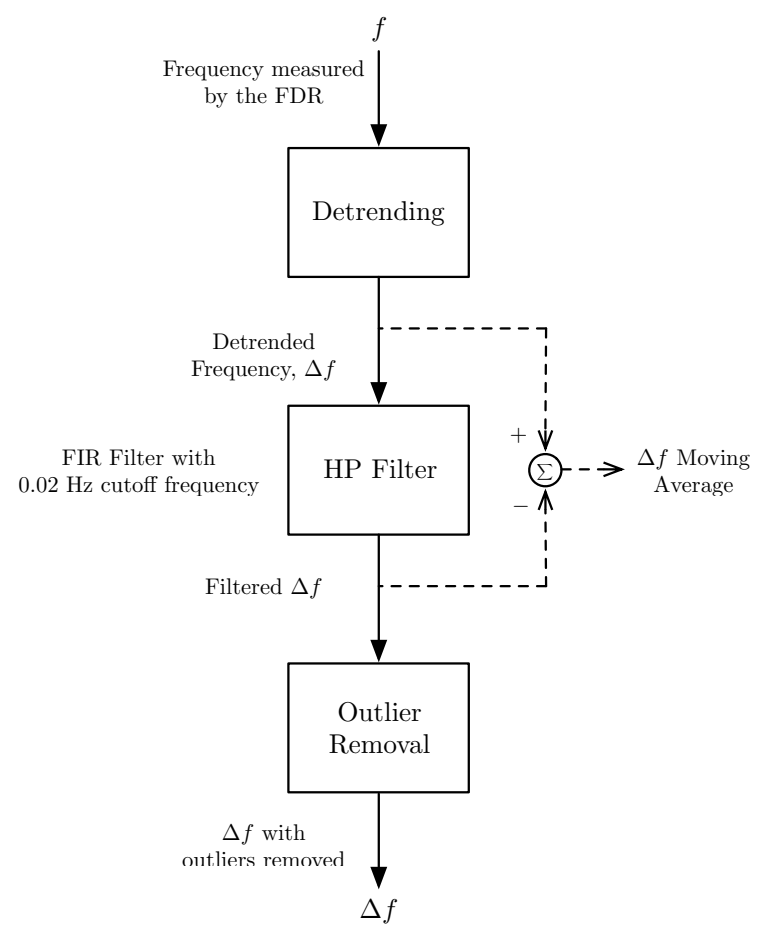

Figure 14. Proposed method for pre-processing FDR data from the Nigerian power system to be used in ambient data analysis

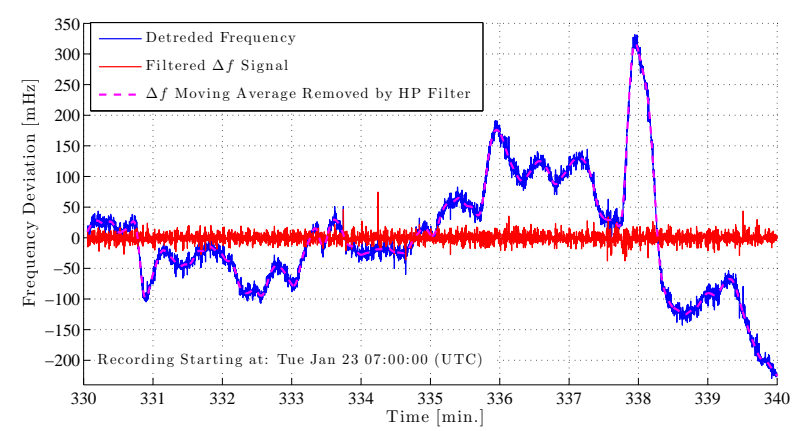

(a) Detrending and Filtering the frequency measurements

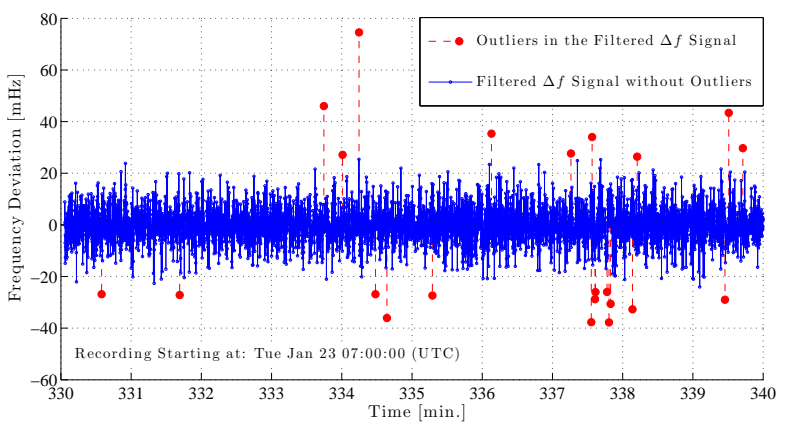

(b) Outlier removal and final $\Delta f$ signal for 10 min. data block

Figure 15. Appling the pre-processing method to a $10 \mathrm{~min}$. data-window from the Tue, Jan. 23, 2007 (N17) data recording.

\section{Mode Frequency Estimates}

For this investigation, ambient data obtained during prolonged recordings resulting in data sets which range from 2 hrs. to $19 \mathrm{hrs}$. are used. Results are provided only for a subset of these data sets. Some of these data sets contained large gaps due to GPS signal loss. In addition, some portions of the data were corrupted by quantization errors, and thus removed for this analysis. The data is segmented in blocks of $10 \mathrm{~min}$., and pre-processed with the method described in the previous section. ${ }^{3}$ To each $10 \mathrm{~min}$. block of pre-processed data the Welch and YW methods are applied, and Welch spectrograms and AR spectrograms are obtained. For the Welch method, 1000 points $(100 \mathrm{sec}$.) data segments are used to calculate the FFTs involved in the averaging portion of the algorithm. To these segments a Hanning window with 50\% overlap is applied. The AR model order used here is $p=30$.

The resulting Welch spectrogram and the AR spectrogram shown in Figs. 16. In the spectograms the red colors represent maximum values and the blue colors represent minimum values of the power spectrum density $[\mathrm{dB}]$. Note that the data sets N16 and N17 forms a continuous set of data recording, however between approximately 8.7 and 10 hrs (shown as a blue band) there were quantization, GPS signal loss, and data drop issues. Therefore, the PSDs for this time frame were not computed and the PSD has been set to zero for all the frequencies within the time range.

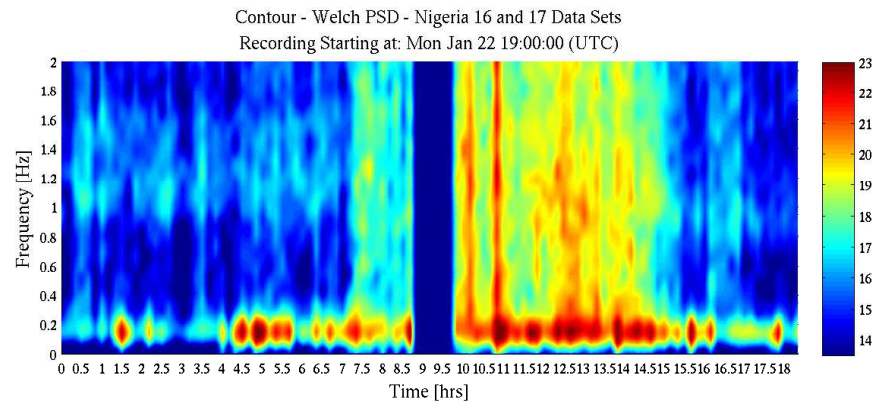

(a) Welch Spectrogram

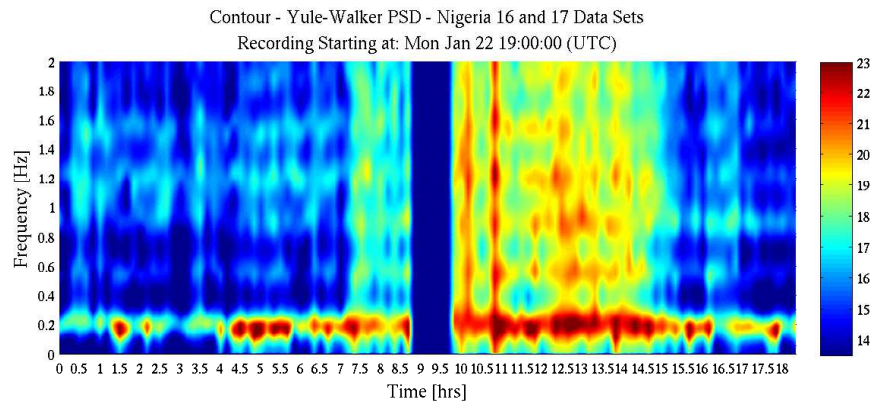

(b) AR Spectrogram

Figure 16. Welch and AR Spectrograms for the $\Delta f$ signal obtained during Mon, Jan 22 ' 07 and Tue., Jan 23 '07 (N16 and N17). The red colors represent maximum values and the blue colors represent minimum values of the power spectrum density [dB]. The time is given in hours in (UTC) starting from 19:00:00 hrs, local time is given in UTC+1 hr.

The Welch spectrogram in Fig. 16a clearly indicates the presence of a dominant inter-area mode with a mean frequency of $0.16 \mathrm{~Hz}$ ranging from $0.12 \mathrm{~Hz}$ to $0.2 \mathrm{~Hz}$. In this spectrogram the variation of the modes with the system stress are quite

\footnotetext{
${ }^{3}$ For convenience, the pre-processed signals are denoted by $\Delta f$.
} 
noticeable with the modes showing large excitation between 10 and 15 hrs., corresponding to 7 - 12 am of Tuesday the 23rd, coinciding with the expected loading of the network for that given time range. Although both the modified (Fig. 16a) and AR (Fig. 16b) spectrograms suggest that two additional modes are present between $0.915-1.025 \mathrm{~Hz}$ and $1.15-1.3 \mathrm{~Hz}$, further data analysis is needed to confirm this indication.

\section{CONCLUSIONS}

This paper has illustrated the application of ambient data analysis techniques for electromechanical mode estimation over multiple hours of data in four different power systems, and provided insight on how to critically analyze the results derived from these techniques. The WECC data analysis, showing the separation of Alberta, illustrates how these techniques are crucial for tracking the changing dynamics of inter-area modes in power networks. In addition, it is important to recognize that, as shown in the data analysis for the US EI and the Nordic Power System, ambient data analysis results should be closely examined for sinusoids or forced oscillations. The presence of these sinusoids will ultimately pose a challenge for mode meter algorithms to provide damping estimates, opening a further research window in this area. Finally, the data analysis of the Nigerian power system data is an interesting example of how to pre-process PMU data for ambient analysis to deal with difficult system dynamics.

\section{APPENDIX}

\section{A. A list of MATLAB and Signal Processing Toolbox commands for Ambient Analysis of PMU Data}

\section{1) Pre-processing:}

a) detrend: used for removing linear trends from a signal.

b) fir1: used for designing low-pass and high-pass filters to remove undesired frequencies from the data.

c) filtfilt: zero-phase digital filtering, used to filter low or high frequency components from the PMU data.

d) interp 1: used for linear interpolation through missing data

2) PSD Estimation:

a) pwelch: estimates the PSD fo a signal using the Welch periodogram.

b) pyulear: implements the Yule-Walker algorithm and computes the PSD of a signal.

c) hamming: creates a hamming window for use with the pwelch function.

3) Construction of Spectrograms:

a) surface: by setting view $(0,90)$, this function can be used to construct spectrograms.

b) contour: used to create spectrograms.

\section{ACKNOWLEDGMENT}

This work is supported by the RPI Power System Research Consortium Industry Members: AEP, FirstEnergy, ISO NE, NYISO, and PJM.

\section{REFERENCES}

[1] A. Hauer, D. Trudnowski, and J. DeSteese, "A Perspective on WAMS Analysis Tools for Tracking of Oscillatory Dynamics," IEEE Power Engineering Society General Meeting, 2007., pp. 1-10, June 2007.

[2] D. Trudnowski and J.W. Pierre, in Inter-area Oscillations in Power Systems: A Nonlinear and Nonstationary Perspective, ser. Power Electronics and Power Systems, A. R. Messina, Ed. Springer, 2009, ch. Signal Processing Methods for Estimating Small-Signal Dynamic Properties from Measured Responses, pp. 1-36.

[3] H. Ghasemi and C. Cañizares, "Confidence intervals estimation in the identification of electromechanical modes from ambient noise," IEEE Transactions on Power Systems, vol. 23, no. 2, pp. 641 -648, May 2008.

[4] H. Ghasemi, C. Cañizares, and A. Moshref, "Oscillatory stability limit prediction using stochastic subspace identification," IEEE Transactions on Power Systems, vol. 21, no. 2, pp. 736 - 745, May 2006.

[5] D. Trudnowski, "Estimating Electromechanical Mode Shape From Synchrophasor Measurements," IEEE Transactions on Power Systems, vol. 23, no. 3, pp. 1188-1195, Aug. 2008.

[6] J. F. Hauer, C. J. Demeure, and L. L. Scharf, "Initial results in Prony analysis of power system response signals," IEEE Transactions on Power Systems, vol. 5, no. 1, pp. 80-89, Feb. 1990.

[7] J. Sanchez-Gasca and J. Chow, "Performance comparison of three identification methods for the analysis of electromechanical oscillations," IEEE Transactions on Power Systems, vol. 14, no. 3, pp. 995-1002, Aug. 1999.

[8] N. Zhou, J. Pierre, and J. Hauer, "Initial results in power system identification from injected probing signals using a subspace method," Power Systems, IEEE Transactions on, vol. 21, no. 3, pp. 1296-1302, Aug. 2006.

[9] J. F. Hauer and J. M. Johnson, A User Guide to PSM Tools: Utilities for Matlab Processing of Power System Response Records, Feb 2001, Prepared for the U.S. Department of Energy Transmission Reliability Program by the Consortium for Electric Reliability Solutions (CERTS). Available online: http://ftp.bpa.gov/outgoing/WAMS\%20Information/.

[10] M. Parashar and J. Mo, "Real Time Dynamics Monitoring System (RTDMS): Phasor Applications for the Control Room," in 42nd Hawaii International Conference on System Sciences, 2009 (HICSS '09), Jan. 2009, pp. 1-11.

[11] J. Proakis and D. Manolakis, Digital Signal Processing Principles, Algorithms, and Applications, 4th ed. Upper Saddle River, New Jersey: Prentice Hall, 2007.

[12] P. Stoica and R. Moses, Spectral Analysis of Signals. Upper Saddle River, New Jersey: Pearson Prentice Hall, 2005.

[13] J. Hauer and R. Cresap, "Measurement and Modeling of Pacific AC Intertie Response to Random Load Switching," IEEE Transactions on Power Apparatus and Systems, vol. PAS-100, no. 1, pp. 353-359, Jan. 1981.

[14] J. Pierre, D. Trudnowski, and M. Donnelly, "Initial Results in Electromechanical Mode Identification from Ambient Data," IEEE Transactions on Power Systems, vol. 12, no. 3, pp. 1245-1251, Aug. 1997. 
[15] J. Pierre and R. F. Kubichek, "Spectral Analysis: Analyzing a Signal Spectrum," Tektronix Application Note, 2002, available online: http://www.tek.com/Measurement/App_Notes/55_15429/eng/.

[16] D. Trudnowski, J. Pierre, N. Zhou, J. Hauer, and M. Parashar, "Performance of Three Mode-Meter Block-Processing Algorithms for Automated Dynamic Stability Assessment," IEEE Transactions on Power Systems, vol. 23, no. 2, pp. 680-690, May 2008.

[17] L. Dosiek, D. Trudnowski, and J. Pierre, "New algorithms for mode shape estimation using measured data," in IEEE Power and Energy Society General Meeting, July 2008, pp. 1-8.

[18] D. Trudnowski, J. Hauer, J. Pierre, W. Litzenberger, and D. Maratukulam, "Using the coherency function to detect large-scale dynamic system modal observability," Proceedings of the 1999 American Control Conference, vol. 4, pp. 2886-2890, 1999.

[19] J. Hauer, N. Bhatt, K. Shah, and S. Kolluri, "Performance of "wams east" in providing dynamic information for the north east blackout of august 14, 2003," in IEEE Power Engineering Society General Meeting, 2004, June 2004, pp. 1685-1690 Vol.2.

[20] L. Vanfretti, T. M. L. Assis, J. H. Chow, L. Dosiek, J. W. Pierre, D. Trudnowski, and Y. Liu, "Data Analysis of the 2/26/08 Florida Blackout " NASPI Work Group Meeting, Sacramento, CA, June 4, 2009, available online:http://www.naspi.org/meetings/workgroup/workgroup.stm.

[21] G. Ledwich and E. Palmer, "Modal estimates from normal operation of power systems," in Power Engineering Society Winter Meeting, 2000. IEEE, vol. 2, 2000, pp. 1527-1531 vol.2.

[22] D. Vowles, M. Gibbard, D. Geddey, and D. Bones, "Benchmark testing methodology for continuous modal-estimation algorithms," in Power \& Energy Society General Meeting, 2009. PES '09. IEEE, July 2009, pp. 1-8.

[23] Z. Zhong, C. Xu, B. Billian, L. Zhang, S.-J. Tsai, R. Conners, V. Centeno, A. Phadke, and Y. Liu, "Power system frequency monitoring network (FNET) implementation," IEEE Transactions on Power Systems, vol. 20, no. 4, pp. 1914-1921, Nov. 2005.

[24] Signal Processing Toolbox ${ }^{\mathrm{TM}} 6$ User's Guide, The MathWorks, Inc., September 2009.

[25] FRCC Event Analysis Team, "FRCC System Disturbance and Underfrequency Load Sheeding Event Report, February 26, 2008 at 1:09 pm," Florida Reliability Coordinating Council, Tech. Rep., October 30, 2008.

[26] F. Tuffner and J. Pierre, "Electromechanical Modal Behavior During a 48 Hour Interval Using Nonparametric Methods," Proceedings of the North American Power Symposium (NAPS), September 2006.

[27] R. P. Schulz, "Power System Dynamics in the Eastern US-Canada Interconnection: Some Experiences," AEP INternal Report, July 2004, available online: http://phasors.pnl.gov.

[28] L. Vanfretti, U. Aliyu, J. H. Chow, and J. Momoh, "System Frequency Monitoring in the Nigerian Power System," Proceedings of the of the IEEE PES General Meeting, 2009. 